\title{
Effect of MHD and Thermal Diffusion on Natural Convection Oscillatory Flow Past Plate with Viscous Heating
}

\author{
Pawan Kumar Sharma ${ }^{1}$ and Chhama Singh ${ }^{2}$ \\ ${ }^{I}$ Department of Applied Mathematics, Amity School of Engineering and Technology, Bijwasan, New Delhi, India \\ ${ }^{2}$ Department of Mathematics, NIIMS University, Jaipur, Rajasthan, India
}

Email:drpawanksharma@yahoo.com,chhama_singh@rediffmail.com

\begin{abstract}
This communication investigates the unsteady two-dimensional oscillatory flow of an incompressible viscous fluid past an infinite vertical, porous flat plate with the effect of magnetic field. It is assume that free stream velocityoscillates in times about a constant mean. Assuming the periodic heat flux at the plate with the effect of viscous dissipation, the set of non-linear coupled differentialequations is solved by regular perturbationtechnique. The approximate solutions are obtained for velocity and temperature field. The effect of various parameters on mean flow velocity, transient velocity, mean skin friction and transient temperature are discussed and shown graphically.
\end{abstract}

Keywords: MHD, viscous dissipation, natural convection, thermal diffusion, oscillatory flow.

\section{Introduction}

From the technological point of view oscillatory flow is always the subject of intensive studies due to its numerous and wide ranging applications. The study of natural convection in a vertical channel with the effect of magnetic field and thermal diffusion is an important subject due to increasing practical applications in industries. Such a study was initiated by Lighthill [1] who studied a two dimensional flow of an incompressible viscous fluid. By assuming that a regular fluctuating flow is superimposed on the mean steady boundary -layer flow, he solved the problem by the momentum method. Stuart [2] extended this idea to study a two-dimensional flow past an infinite vertical porous flat plate when the free stream velocity oscillate in time about a constant mean, where he assumed that there is no heat transfer between the plate and the fluid in deriving the temperature field, which is only one of the possible cases of physical situation. Soundalgekar [3-4] discussed the other case of physical situation, that is when the difference between the plate temperature and the free stream temperature isapparently large so as to cause the free convection currents to the flow in the boundary layer and the free stream velocity oscillates in time about a constant mean in the direction of the flow. Since the flow is very slow and hence viscous dissipative effects are negligible. In the case of fluid with high Prandtl number, viscous dissipative heat is always present even in slow motion. In case of unsteady free convective flows Soundalgekar [5] studied the effects of viscous dissipation on the flow past an infinite vertical porous plate. It was assumed that the plate temperature oscillates in such a way that its amplitude is small. The laminar free convection from a vertical plate has been investigated by Martynenko et al.[6] . Harris et al.[7] studied free convection from a vertical plate through a porous media. Magnetic fields influence many natural and man-made flows. They are routinely used in industry to heat, pump, stir and levitate liquid metals. The terrestrial magnetic field, which is maintained by fluid, motion in the earth's core, the solar magnetic fields, which generates sunspots and solar flares, and the galactic field which influences the formation of stars. The flow problems of an electrically conducting fluid under the influence of magnetic field have attracted the interest of many authors in view of its applications to geophysics, astrophysics, engineering, and to the boundary layer control in the field of aerodynamics. On the other hand in view of the increasing technical applications using Magnetohydrodynamics effect, it is desirable to extend many of the available viscous hydrodynamic 
solution to include the effects of magnetic field for those cases when the viscous fluid is electrically conducting. Rossow [8], Greenspan and Carrier [9] have studied extensively the hydromagnetic effects on the flow past a plate with or without injection/suction. The hydromagnetic channel flow and temperature field was investigated by Attia and Kotab [10]. Hossain et al. [11] have studied the MHD free convection flow when the surface kept at oscillating surface heat flux. Recently Sharma et al. [12] have studied the unsteady free convection oscillatory flow between parallel plates through porous medium assuming periodic temperature variations. Boundary layer flows of fluids of small electrical conductivity are important particularly in the field of aeronautical engineering. Therefore the object of the present paper is to study the effects of the magnetic field and oscillatory thermal diffusion on the unsteady flow of a viscous, incompressible and electrically conducting fluid past vertical porous flat plate with oscillatory free stream velocity and viscous dissipative effect at the plate is also considered.

\section{Formulation of the Problem}

We consider the unsteady flow of a viscous incompressible fluid past infinite vertical porous flat plate, with a free stream velocity that oscillates in time about a constant mean. We take $\mathrm{x}^{*}$-axis along the moving vertical plate in the vertically upward direction which is the direction of flow and $\mathrm{z}^{*}$-axis is taken normal to the plate. We consider the free-stream velocity distribution of the form:

$$
\text { (1) } \left.U^{*}\left(t^{*}\right)=U_{0}\left(1+\varepsilon e^{i \omega^{*} t^{*}}\right), \quad w^{*}=-w_{0} \text { ( cons } \tan t \text { suction velocity }\right)
$$

where $U_{\mathrm{o}}$ is the mean constant free-stream velocity, $\omega^{*}$ is the frequency of oscillations and $t^{*}$ is the time. The basic equation of magnetofluiddynamics and conventional fluiddynamics are different by only additional force term due to electromagnetic field. The Maxwell's equations have to be satisfied in the entire field. In order to derive the basic equations for the problem under consideration, the following assumptions are made:

1) The flow is steady and laminar and the magnetic field is applied perpendicular to the plate.

2) The fluid under consideration is viscous, incompressible and finitely conducting with constant physical properties.

3) The magnetic Reynolds number is taken to be small enough so that the induced magnetic field is neglected.

4) Hall Effect, electrical and polarization effects are neglected.

The equations governing the problem are:

$$
\text { (2) } \frac{\partial u^{*}}{\partial t^{*}}+w^{*} \frac{\partial u^{*}}{\partial z^{*}}=\frac{\partial U^{*}}{\partial t^{*}}+v \frac{\partial^{2} u^{*}}{\partial z^{* 2}}+g \beta\left(T^{*}-T_{\infty}^{*}\right)-\frac{(\vec{J} \times \vec{B})}{\rho}
$$

where the fourth term on the right hand side of equation (2) is the Lorentz force due to magnetic field $\overrightarrow{\mathrm{B}}$, and is given by

(3) $\vec{J} \times \vec{B}=\sigma(\vec{v} \times \vec{B}) \times \vec{B}$

The equation (2) becomes

$$
\text { (4) } \frac{\partial u^{*}}{\partial t^{*}}+w^{*} \frac{\partial u^{*}}{\partial z^{*}}=\frac{\partial U^{*}}{\partial t^{*}}+v \frac{\partial^{2} u^{*}}{\partial z^{* 2}}+g \beta\left(T^{*}-T_{\infty}^{*}\right)-\frac{\sigma B^{2}}{\rho}\left(u^{*}-U^{*}\right)
$$

The energy equation is:

$$
\frac{\partial T^{*}}{\partial t^{*}}+w^{*} \frac{\partial T^{*}}{\partial z^{*}}=\frac{\kappa}{\rho C_{p}} \frac{\partial^{2} T^{*}}{\partial z^{*}}+\frac{v}{C_{p}}\left(\frac{\partial u^{*}}{\partial z^{*}}\right)^{2}
$$

where $\mathrm{u}^{*}, \mathrm{U}^{*}, v, \mathrm{~g}, \beta, \mathrm{k}, \mathrm{T}^{*}, \mathrm{~T}_{\infty}{ }^{*}, \mathrm{C}_{\mathrm{p}}$ are respectively, velocity, free-stream velocity, kinematic viscosity, gravity, volumetric coefficient of thermal expansion, thermal conductivity, temperature of fluid in the boundary layer, temperature of the plate at in free stream, and specific heat at constant pressure of the fluid. The $(*)$ stands for dimensional quantities. The boundary conditions of the fluid motion are :

$$
z^{*}=0: \quad u^{*}=0, \frac{\partial T^{*}}{\partial z^{*}}=-\frac{q_{w}^{*}}{\kappa}\left(1+\varepsilon e^{i \omega^{*} t^{*}}\right)
$$


$z^{*} \rightarrow \infty: u^{*}=U_{0}\left(1+\varepsilon e^{\imath w^{*} t^{*}}\right), T^{*}=T_{\infty}^{*}$.

Introducing the following non - dimensional quantities

$z=\frac{z^{*} w_{0}}{v}, u=u^{*} / U_{0}, U=U^{*} / U_{0}, t=\frac{w_{0}{ }^{2} t^{*}}{v}, \omega=\omega^{*} v / U_{0}{ }^{2}$,

$\theta=\frac{\kappa U_{0}\left(T^{*}-T_{\infty}{ }^{*}\right)}{{q_{w}{ }^{*} v} v}, \operatorname{Gr}($ Grassoff number $)=\frac{g \beta v^{2}{q_{w}}^{*}}{\kappa U_{0}{ }^{2} w_{0}{ }^{2}}$,

$\alpha($ thermal diffusivity $)=\frac{\kappa}{\rho C_{p}}, \operatorname{Er}($ Ec ker $t$ number $)=\frac{U_{0}{ }^{3} \kappa}{C_{p} v q_{w}{ }^{*}}$,

$M($ Hartmann Number $)=\sqrt{\frac{\sigma B^{2} v}{\rho w_{0}^{2}}}, \operatorname{Pr}(\operatorname{Pr}$ andtl number $)=v / \alpha$

The equations (4) and (5) become

$$
\begin{aligned}
& \frac{\partial u}{\partial t}-\frac{\partial u}{\partial z}=\frac{\partial U}{\partial t}+\frac{\partial^{2} u}{\partial z^{2}}+G r \theta-M^{2}(u-U) \\
& \frac{\partial \theta}{\partial t}-\frac{\partial \theta}{\partial z}=\frac{1}{\operatorname{Pr}} \frac{\partial^{2} \theta}{\partial y^{2}}+E c\left(\frac{\partial u}{\partial z}\right)^{2}
\end{aligned}
$$

with corresponding boundary conditions

\section{Solution of the Problem}

$$
\left.\begin{array}{rl}
z=0: u & =0, \frac{\partial \theta}{\partial z}=-\left(1+\varepsilon e^{i t}\right) \\
z & \rightarrow \infty: u=1+\varepsilon e^{i \omega t}, \quad \theta=0
\end{array}\right\}
$$

Since the amplitudes of the free-stream velocity and temperature variation $\varepsilon(<<1)$ is very small, we now assume the solutions of the following form :

$$
\left.\begin{array}{l}
u(z, t)=u_{0}(z)+\varepsilon u_{1}(z) e^{i t}+\ldots \ldots \ldots \ldots, \\
\theta(z, t)=\theta_{0}(z)+g \theta_{1}(z) e^{i t}+\ldots \ldots \ldots \ldots . .
\end{array}\right\}
$$

and for the free-stream velocity

$$
\mathrm{U}=1+\varepsilon \mathrm{e}^{\mathrm{it}} .
$$

Substituting equations (10) and (11) in equations (7) and (8), comparing the coefficients of identical power of $\varepsilon$ and neglecting those of $\varepsilon^{2}$, we get following equations

$$
\begin{aligned}
& u_{0}^{\prime \prime}+u_{0}{ }^{\prime}-M^{2} u_{0}=-G r \theta_{0}-M^{2}, \\
& \theta_{0}^{\prime \prime}+\operatorname{Pr} \theta_{0}^{\prime}=-E c \operatorname{Pr}\left(u_{0}^{\prime}\right)^{2}, \\
& u_{1}^{\prime \prime}+u_{1}^{\prime}-\left(i \omega+M^{2}\right) u_{1}=-G r \theta_{1}-\left(i \omega+M^{2}\right), \\
& \theta_{1}^{\prime \prime}+\operatorname{Pr} \theta_{1}^{\prime}-i \omega \operatorname{Pr} \theta_{1}=-2 E c \operatorname{Pr} u_{o}^{\prime} u_{1}^{\prime} .
\end{aligned}
$$

with the corresponding boundary conditions :

$$
\left.\begin{array}{rl}
z=0: u_{0}=0, u_{1}=0, \frac{\partial \theta_{0}}{\partial z}=-1, \frac{\partial \theta_{1}}{\partial z}=-1 \\
z \rightarrow \infty: u_{0}=1, u_{1}=1, \theta_{0}=0, \theta_{1}=0
\end{array}\right\}
$$


where primes denotes differentiation with respect to $\mathrm{z}$. To solve the coupled non-linear equations (12) to (15), we now assume that the heat due to viscous dissipation is superimposed on the motion. Mathematically this can be achieved by expanding the velocity and temperature terms in power of Ec( Eckert Number). In the case of incompressible fluids, Ec is always very small. We now assume

$$
\begin{aligned}
& u_{0}(z)=u_{01}(z)+E c u_{02}(z)+O\left(E c^{2}\right) \\
& u_{1}(z)=u_{11}(z)+E c u_{12}(z)+O\left(E c^{2}\right) \\
& \theta_{0}(z)=\theta_{01}(z)+E c \theta_{02}(z)+O\left(E c^{2}\right) \\
& \theta_{0}(z)=\theta_{11}(z)+E c \theta_{12}(z)+O\left(E c^{2}\right)
\end{aligned}
$$

Substituting (17) in (12) to (15), equating to zero the coefficient of different powers of Ec and neglecting the term of $\mathrm{O}\left(\mathrm{Ec}^{2}\right)$, we obtain the following set of equations with corresponding boundary conditions:

$$
\begin{aligned}
& u_{01 z z}+u_{01 z}-M^{2} u_{01}=-G r \theta_{01}-M^{2} \\
& u_{02 z z}+u_{02 z}-M^{2} u_{02}=-G r \theta_{02} \\
& u_{11 z z}+u_{11 z}-\left(M^{2}+\imath \omega\right) u_{11}=-G r \theta_{11}-\left(M^{2}+\imath \omega\right) \\
& u_{12 z z}+u_{12 z}-\left(M^{2}+\imath \omega\right) u_{12}=-G r \beta_{12} \\
& \left.z=0: \quad u_{01}=0, u_{02}=0, u_{11}=0, u_{12}=0\right\} \\
& z \rightarrow \infty: \quad u_{01}=1, u_{02}=0, u_{11}=1, u_{12}=0 \\
& \theta_{01 z z}+\operatorname{Pr} \theta_{01 z}=0 \\
& \theta_{02 z z}+\operatorname{Pr} \theta_{02 z}=-\operatorname{Pr}\left(u_{01 z}\right)^{2} \\
& \theta_{11 z z}+\operatorname{Pr} \theta_{11 z}-\imath \omega \operatorname{Pr} \theta_{11}=0 \\
& \theta_{12 z z}+\operatorname{Pr} \theta_{12 z}-\imath \omega \operatorname{Pr} \theta_{12}=-2 \operatorname{Pr} u_{01 z} u_{11 z} \\
& z=0: \quad \theta_{01 z}=-1, \theta_{02 z}=0, \quad \theta_{11 z}=-1, \theta_{12 z}=0 \\
& \left.z \rightarrow \infty: \quad \theta_{01}=0, \quad \theta_{02}=0, \quad \theta_{11}=0, \quad \theta_{12}=0\right\}
\end{aligned}
$$

Solving equations (18) to (21) and (23) to (26) under the corresponding boundary conditions (22) and (27), we get:

$$
\begin{aligned}
& \theta_{01}(z)=\frac{1}{\operatorname{Pr}} e^{-\operatorname{Pr} z}, \\
& \begin{array}{l}
\theta_{02}(z)=\frac{1}{\operatorname{Pr}}\left[2 n_{2} n_{4}+2 n_{5} \operatorname{Pr}-n_{6} n_{2}-n_{6} \operatorname{Pr}\right] e^{-\operatorname{Pr} z} \\
\quad-n_{4} e^{-2 n_{2} z}-n_{5} e^{-2 \operatorname{Pr} z}+n_{6} e^{-\left(n_{2}+\operatorname{Pr}\right) z}, \\
\theta_{11}(z)=\frac{1}{n_{1}} e^{-n_{1} z}, \\
\theta_{12}(z)=n_{18} e^{-n_{1} z}-n_{9} e^{-\left(n_{2}+n_{7}\right) z}+n_{10} e^{-\left(n_{1}+n_{2}\right) z}+n_{11} e^{-\left(n_{2}+n_{7}\right) z}+n_{12} e^{-\left(n_{2}+\operatorname{Pr}\right) z} \\
-n_{13} e^{-\left(n_{1}+\operatorname{Pr}\right) z}-n_{14} e^{-\left(n_{7}+\operatorname{Pr}\right) z}+n_{15} e^{-\left(n_{2}+n_{7}\right) z}-n_{16} e^{-\left(n_{1}+n_{2}\right) z}-n_{17} e^{-\left(n_{2}+n_{7}\right) z}, \\
u_{01}(z)=n_{3}\left(e^{-n_{2} z}-e^{-\operatorname{Pr} z}\right)+e^{-n_{2} z}+1, \\
u_{02}(z)=b_{5} e^{-n_{2} z}-b_{1} e^{-\operatorname{Pr} z}+b_{2} e^{-2 n_{2} z}+b_{3} e^{-2 \operatorname{Pr} z}-b_{4} e^{-\left(n_{2}+\operatorname{Pr}\right) z}, \\
u_{11}(z)=n_{8}\left(e^{-n_{7} z}-e^{-n_{1} z}\right)-e^{-n_{7} z}+1,
\end{array}
\end{aligned}
$$




$$
\begin{aligned}
& u_{12}(z)=n_{29} e^{-n_{7} z}-n_{19} e^{-n_{1} z}+n_{20} e^{-\left(n_{2}+n_{7}\right) z}-n_{21} e^{-\left(n_{2}+n_{1}\right) z}-n_{22} e^{-\left(n_{2}+n_{7}\right) z} \\
& -n_{23} e^{-\left(n_{2}+\operatorname{Pr}\right) z}+n_{24} e^{-\left(n_{1}+\operatorname{Pr}\right) z}+n_{25} e^{-\left(\operatorname{Pr}+n_{7}\right) z}-n_{26} e^{-\left(n_{7}+n_{2}\right) z}+n_{27} e^{-\left(n_{2}+n_{1}\right) z}+n_{28} e^{-\left(n_{2}+n_{7}\right) z}
\end{aligned}
$$

where:

$$
\begin{aligned}
& n_{1}=\frac{1}{2}\left[\operatorname{Pr}+\sqrt{\operatorname{Pr}^{2}+4 i \omega \operatorname{Pr}}\right], n_{2}=\frac{1}{2}\left[1+\sqrt{1+4 M^{2}}\right] \text {, } \\
& n_{3}=\frac{G r}{\operatorname{Pr}\left(\operatorname{Pr}^{2}-\operatorname{Pr}-M^{2}\right)}, n_{4}=\frac{\operatorname{Pr} n_{2}^{2}\left(n_{3}-1\right)^{2}}{4 n_{2}^{2}-2 n_{2} \operatorname{Pr}}, n_{5}=\frac{n_{3}^{2} \operatorname{Pr}}{2}, n_{6}=\frac{2 \operatorname{Pr} n_{2} n_{3}\left(n_{3}-1\right)}{n_{2}^{2}+n_{2} \operatorname{Pr}}, \\
& n_{7}=\frac{1}{2}\left[1+\sqrt{1+4\left(M^{2}+i \omega\right)}\right], n_{8}=\frac{G r}{n_{1}\left(n_{1}^{2}-n_{1}-M^{2}-\imath \omega\right)} \\
& n_{9}=\frac{2 \operatorname{Pr} n_{2} n_{3} n_{7} n_{8}}{\left(n_{2}+n_{7}\right)^{2}-\operatorname{Pr}\left(n_{2}+n_{7}\right)-\imath \omega \operatorname{Pr}}, n_{10}=\frac{2 \operatorname{Pr} n_{2} n_{3} n_{7}}{\left(n_{2}+n_{7}\right)^{2}-\operatorname{Pr}\left(n_{2}+n_{7}\right)-\imath \omega \operatorname{Pr}} \\
& n_{11}=\frac{2 \operatorname{Pr} n_{2} n_{3} n_{1} n_{8}}{\left(n_{1}+n_{2}\right)^{2}-\operatorname{Pr}\left(n_{1}+n_{2}\right)-\imath \omega \operatorname{Pr}}, n_{12}=\frac{2 \operatorname{Pr}^{2} n_{8} n_{3} n_{7}}{\left(\operatorname{Pr}+n_{7}\right)^{2}-\operatorname{Pr}\left(\operatorname{Pr}+n_{7}\right)-\imath \omega \operatorname{Pr}}, \\
& n_{13}=\frac{2 \operatorname{Pr}^{2} n_{3} n_{1} n_{8}}{\left(n_{1}+\operatorname{Pr}\right)^{2}-\operatorname{Pr}\left(n_{1}+\operatorname{Pr}\right)-\imath \omega \operatorname{Pr}}, n_{14}=\frac{2 \operatorname{Pr}^{2} n_{3} n_{7}}{\left(\operatorname{Pr}+n_{7}\right)^{2}-\operatorname{Pr}\left(\operatorname{Pr}+n_{7}\right)-\imath \omega \operatorname{Pr}}, \\
& n_{15}=\frac{2 \operatorname{Pr} n_{2} n_{7} n_{8}}{\left(n_{2}+n_{7}\right)^{2}-\operatorname{Pr}\left(n_{2}+n_{7}\right)-\imath \omega \operatorname{Pr}}, n_{16}=\frac{2 \operatorname{Pr} n_{2} n_{1} n_{8}}{\left(n_{1}+n_{2}\right)^{2}-\operatorname{Pr}\left(n_{1}+n_{2}\right)-\imath \omega \operatorname{Pr}}, \\
& n_{17}=\frac{2 \operatorname{Pr} n_{2} n_{7}}{\left(n_{2}+n_{7}\right)^{2}-\operatorname{Pr}\left(n_{2}+n_{7}\right)-\imath \omega \operatorname{Pr}} \text {, } \\
& n_{18}=\frac{1}{n_{1}}\left[n_{9}\left(n_{2}+n_{7}\right)-n_{10}\left(n_{1}+n_{2}\right)-n_{11}\left(n_{2}+n_{7}\right)-n_{12}\left(\operatorname{Pr}+n_{7}\right)+n_{13}\left(n_{1}+\operatorname{Pr}\right)\right. \\
& \left.+n_{14}\left(\operatorname{Pr}+n_{7}\right)-n_{15}\left(n_{2}+n_{7}\right)+n_{16}\left(n_{1}+n_{2}\right)+n_{17}\left(n_{2}+n_{7}\right)\right], \\
& n_{19}=\frac{G r n_{18}}{n_{1}^{2}-n_{1}-M^{2}-\imath \omega}, \quad n_{20}=\frac{G r n_{9}}{\left(n_{2}+n_{7}\right)^{2}-\left(n_{2}+n_{7}\right)-M^{2}-\imath \omega}, \\
& n_{21}=\frac{G r n_{10}}{\left(n_{1}+n_{2}\right)^{2}-\left(n_{1}+n_{2}\right)-M^{2}-\imath \omega} \quad, \quad n_{22}=\frac{G r n_{11}}{\left(n_{2}+n_{7}\right)^{2}-\left(n_{2}+n_{7}\right)-M^{2}-\imath \omega}, \\
& n_{23}=\frac{G r n_{12}}{\left(\operatorname{Pr}+n_{2}\right)^{2}-\left(\operatorname{Pr}+n_{2}\right)-M^{2}-\imath \omega} \quad, \quad n_{24}=\frac{G r n_{13}}{\left(\operatorname{Pr}+n_{1}\right)^{2}-\left(\operatorname{Pr}+n_{1}\right)-M^{2}-\imath \omega} \text {, } \\
& n_{25}=\frac{G r n_{14}}{\left(\operatorname{Pr}+n_{7}\right)^{2}-\left(\operatorname{Pr}+n_{7}\right)-M^{2}-\imath \omega} \quad, \quad n_{26}=\frac{G r n_{15}}{\left(n_{2}+n_{7}\right)^{2}-\left(n_{2}+n_{7}\right)-M^{2}-\imath \omega} \text {, } \\
& n_{27}=\frac{G r n_{16}}{\left(n_{1}+n_{2}\right)^{2}-\left(n_{1}+n_{2}\right)-M^{2}-\imath \omega} \quad, \quad n_{28}=\frac{G r n_{17}}{\left(n_{2}+n_{7}\right)^{2}-\left(n_{2}+n_{7}\right)-M^{2}-\imath \omega}, \\
& b_{1}=\frac{G r\left(n_{4}+n_{5}-n_{6}\right)}{\operatorname{Pr}^{2}-\operatorname{Pr}-M^{2}}, b_{2}=\frac{G r n_{4}}{4 n_{2}^{2}-2 n_{2}-M^{2}}, b_{3}=\frac{G r n_{5}}{4 \operatorname{Pr}^{2}-2 \operatorname{Pr}-M^{2}} \text {, } \\
& b_{4}=\frac{G r n_{6}}{\left(\operatorname{Pr}+n_{2}\right)^{2}-\left(\operatorname{Pr}+n_{2}\right)-M^{2}}, b_{5}=b_{1}-b_{2}-b_{3}+b_{4} \text {. }
\end{aligned}
$$

\section{Result and Discussions}

In order to point out the effect of Hartmann Number (M), Eckert number (Ec), Grashoff Number (Gr) and Prandtl number $(\mathrm{Pr})$, on the mean velocity, mean skin-friction, transient velocity and temperature when the moving plate is subjected to oscillating free-stream velocity and fluctuating heat flux with the effect of viscous dissipation function, the following discussions are set out.

\section{(a) Mean flow :}

The mean flow velocity component for $\mathrm{Ec}=0.01$ is presented in Fig. 1 . It is observed from the figure that the mean flow velocity increases with Grashoff number Gr and magnetic field parameter M near the plate and become constant far away from the plate. The mean flow velocity decreases by increasing Prandtl number Pr. Physically this is true because the increase in Prandtl number is due to increase in the viscosity 
of the fluid, which makes the fluid thick and hence a decrease in the velocity of the fluid in the vicinity of the plate and become constant later.

After knowing the mean flow velocity field, it is important to know the effects of magnetic field and Grashoff number on mean- skin friction at the plate. It is given by :

$$
\tau *=\left(\frac{d u^{*}}{d z^{*}}\right)_{z^{*}=0},
$$

and in non - dimensional form it is given by :

$$
\tau=\frac{\tau^{*} b}{\mu U_{0}}=\left(\frac{\partial u}{\partial z}\right)_{z=0}=\left(\frac{\partial u_{0}}{\partial z}\right)_{z=0}+\varepsilon\left(\frac{\partial u_{1}}{\partial z}\right)_{z=0} e^{i t} .
$$

Denoting the mean skin friction by

$$
\tau_{m}=\left(\frac{d u_{0}}{d z}\right)_{z=0}, \tau_{m}=n_{3}\left(\operatorname{Pr}-n_{2}\right)+n_{2}+E c\left[-n_{2} b_{5}+b_{1} \operatorname{Pr}+-2 n_{2} b_{2}-2 \operatorname{Pr} b_{3}+\left(n_{2}+\operatorname{Pr}\right) b_{4}\right]
$$

The mean skin friction profile for $\mathrm{Ec}=0.01$ is presented in Fig.2. It is observed that mean skin friction increases with increasing Gr ( Grashoff number) and $\operatorname{Pr}$ ( Prandtl number ) in the same manner. The mean skin friction increases continuously with increasing M., the magnitude is less for small values of $\mathrm{M}$.

The mean temperature profile is presented in Fig.3. It is observed that mean temperature increases with $\mathrm{M}$ near the plate and become constant as we go away from the plate. It is also observed that mean temperature increases slightly with $\mathrm{Ec}$ ( Eckert number ). Furthermore for higher value of $\mathrm{M}$ the mean temperature decreases rapidly with distance from the plate.

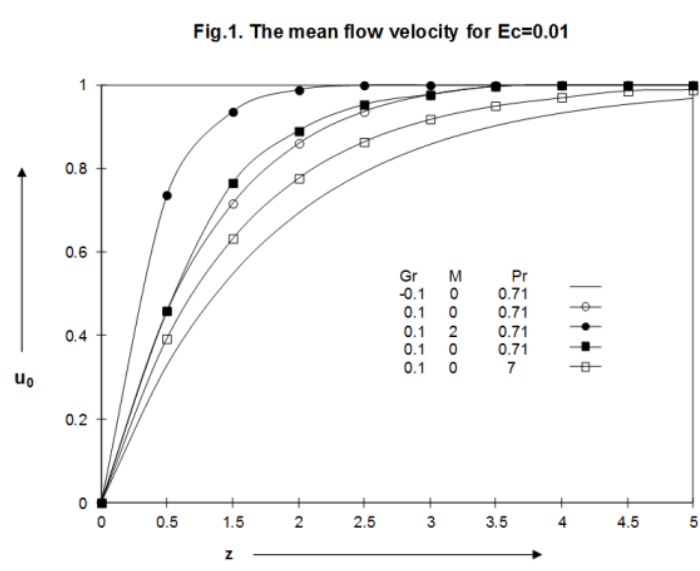

Fig.3. Mean temperature profile

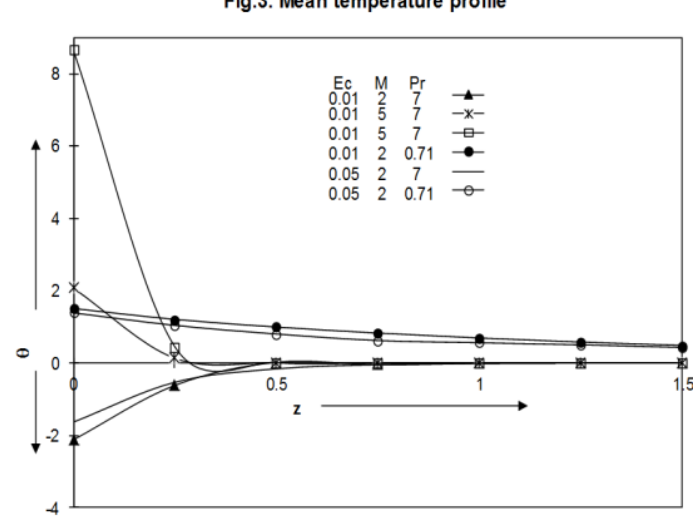

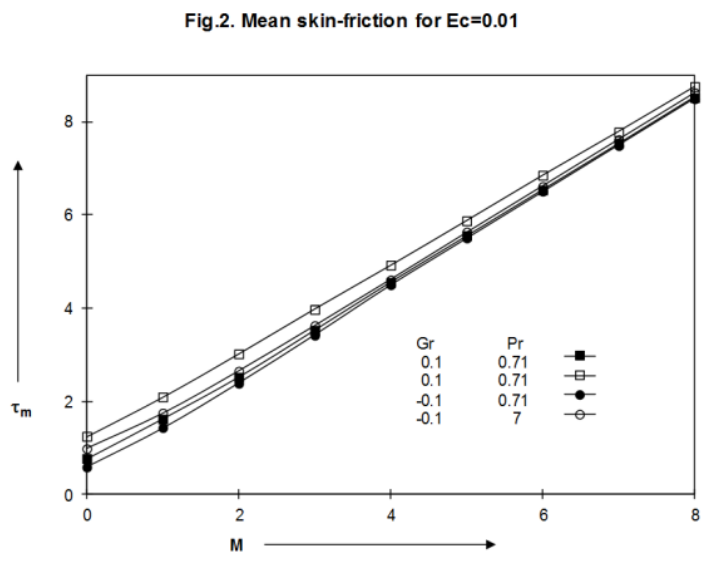

Fig.4.Transient velocity profile for $\mathrm{Pr}=0.71$ (air), $\mathrm{Ec}=-0.01$ $\varepsilon=0.2$ and $t=\pi / 2 \omega$

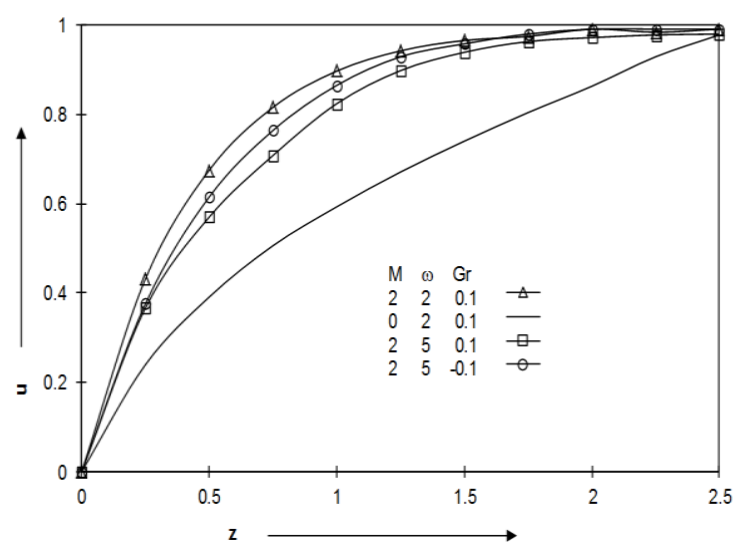



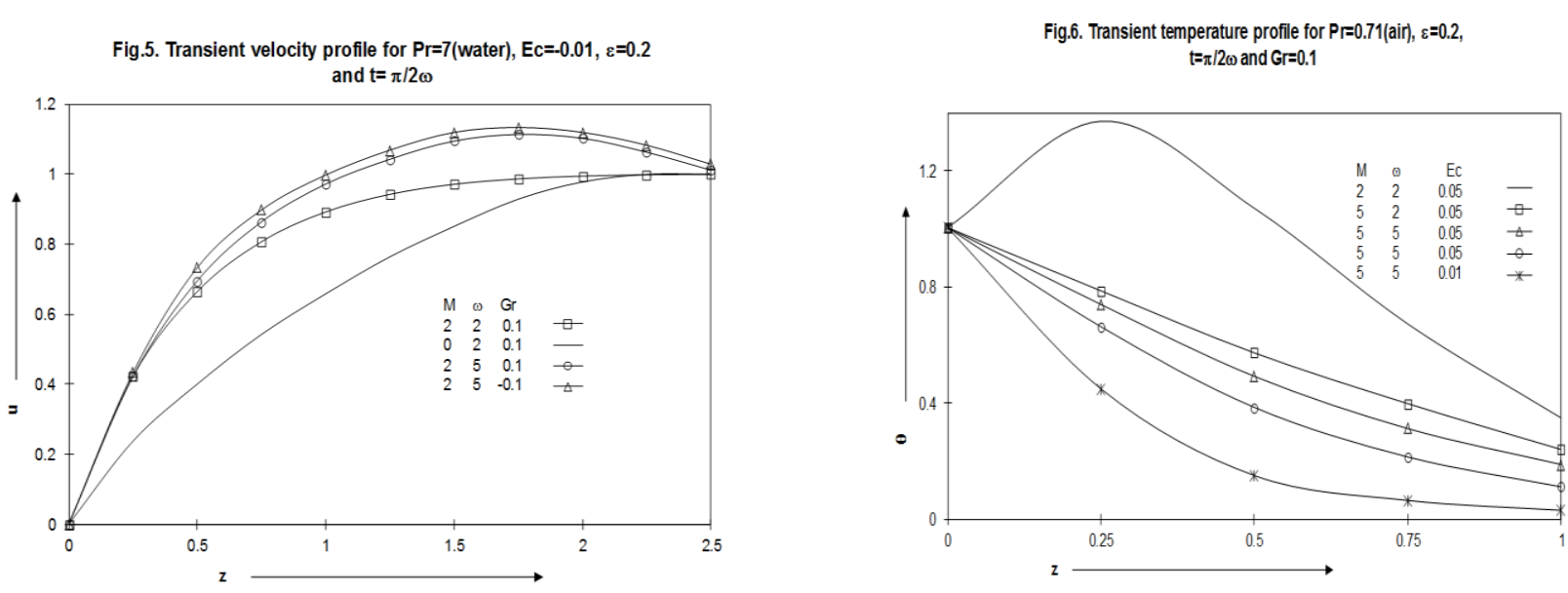

(b)

\section{Unsteady flow:}

The velocity and temperature field can now be expressed in terms of fluctuating parts as follows

$u(z, t)=u_{0}(z)+\varepsilon e^{i t}\left(N_{r}+i N_{i}\right) \quad \theta(z, t)=\theta_{0}(z)+\varepsilon e^{i t}\left(Q_{r}+i Q_{i}\right)$

where: $N_{r}+i N_{i}=u_{1}(z), \quad Q_{r}+i Q_{i}=\theta_{1}(z)$

We can now write expressions for transient velocity and temperature for $t=\pi / 2 \omega$, as follows

$u(z, \pi / 2 \omega)=u_{0}(z)-\varepsilon N_{i}, \quad \theta(z, \pi / 2 \omega)=\theta_{0}(z)-\varepsilon Q_{i}$

For small value of $\varepsilon=0.2$ and $E c=-0.01$ the transient velocity profile for air $(\operatorname{Pr}=0.71)$ is shown in Fig.4. The graph reveals that the transient velocity increases rapidly with $\mathrm{M}$ and angular velocity $(\omega)$. It is also observed that the velocity increases with decreasing Gr. Physically it is true because free convection resist the flow. The transient velocity for small value of $\varepsilon=0.2$ and $\mathrm{Ec}=-0.01$ for water $(\operatorname{Pr}=7)$ is presented in Fig.5. It is observed that it increases with increasing angular velocity $(\omega)$ and reverse effect is observed for Gr. Also velocity increases near the plate and become constant as we go away from the plate. The transient temperature is presented for $\varepsilon=0.2$ and $E c=-0.01$ for air $(\operatorname{Pr}=0.71)$ in Fig. 6 . It is observed that it increases with increasing Ec, while reverse effect is observed for $\omega$. Physically it is true because due to viscous dissipation heat generated by friction of fluid layers lead to rise in temperature. It is also observes from the figure that temperature decreases with increasing $\mathrm{M}$. There is slight decrease in temperature with the plate distance. For lower value of $\mathrm{M}$, the transient temperature increases near the plate and attains maximum value then it decreases with the plate distance.

\section{Conclusions}

Mean and transient velocity increases near the plate and becomes constant far away from the plate. Mean and transient velocity increases with increasing the magnetic field parameter, while reverse effect is observed for Gr. Mean temperature increases with Gr ( Grashoff number). Transient temperature slightly decreases with the distance from plate. It is interesting to observe that transient temperature increases near the plate for small value of $\mathrm{M}$.

\section{References}

[1] M.J. Lighthill; The response of laminar skin friction and heat transfer to fluctuations in the stream velocity, Proceeding of the royal society of London, A224, 1-23 (1954).

[2] T.J. Stuart; A solution of the Navier Stokes and Energy equations illustrating the response of skin-friction and temperature of an infinite plate thermometer to fluctuating in the free stream velocity, Proceeding of the royal society of London, A231, 116-130 (1955).

[3] V.M. Soundalgekar; Free convection effects on the oscillatory flow past an infinite, vertical porous plate with constant suction I, Proceeding of the royal society of London, A333, 25-36 (1973). 
[4] V.M. Soundalgekar; Free convection effects on the oscillatory flow past an infinite, vertical porous plate with constant suction II, Proceeding of the royal society of London, A333, 37-42 (1973).

[5] V.M. Soundalgekar; Viscous dissipation effects on unsteady free convective flow past an infinite vertical porous plate with constant suction, Int. J. Heat Mass Transfer, 15, 1253-1261(1972).

[6] O.G. Martynenko, A.A. Berezovsky and Yu. A. Sokovishin ; Laminar free convection from a vertical plate, Int. J. Heat Mass Transfer, 27, 869-881(1984).

[7] S.D. Harris, D.B. Ingham and I. Pop; Free convection from a vertical plate in a porous medium subjected to a sudden change in surface temperature, Int. Comm. Heat Mass Transfer, 24, 543-552 (1997).

[8] V. J. Rossow, On flow of electrically conducting fluids over a flat plate in the presence of a transverse magnetic field, N. A. C. A., Rept., 1358 (1958).

[9] H. P. Greenspan and G. F. Carrier, The MHD flow past a flat plate, J. Fluid Mech., 6, 77-96 (1959).

[10]H. A. Attia , N. A. Kotab, MHD flow between two parallel plates with heat ransfer, Acta Mech. ,117, 215-220 (1996).

[11] M. A. Hossain , S. K. Das , I. Pop, Heat transfer response of MHD free convection flow along a vertical plate to surface temperature oscillation, Int. J. Non-linear Mech., 33, 541-553(1998).

[12] Sharma Pawan Kumar, Sharma, Bhupendra Kumar and Chaudhary, R.C., Unsteady free convection oscillatory Couette flow through a porous medium with periodic wall temperature, Tamkang Journal of Mathematics, 38, 93-102 (2007). 